\title{
CT Angiography of the Head in Extracorporeal Membrane Oxygenation
}

\author{
(D). Acharya, (D)A.G. Rajamohan, (DM.R. Skalski, (DM. Law, (DP. Kim, and DW. Gibbs
}

\begin{abstract}
SUMMARY: Extracorporeal membrane oxygenation is an artificial cardiopulmonary bypass technique used to support patients with severe pulmonary failure or both pulmonary and cardiac failure. The hemodynamic changes produced by extracorporeal membrane oxygenation affect the appearance of CTA of the head images, often confounding interpretation if the correct history and understanding of extracorporeal membrane oxygenation are not known. This technical report describes the principles of extracorporeal membrane oxygenation, techniques to optimize intracranial CTA imaging, and pitfalls.
\end{abstract}

ABBREVIATIONS: ECMO = extracorporeal membrane oxygenation; $\mathrm{VA}=$ venoarterial; $\mathrm{VV}=$ venovenous

$\mathrm{E}$ tracorporeal membrane oxygenation (ECMO) is an artificial cardiopulmonary bypass technique that is used to support patients with severe pulmonary failure or combined pulmonary and cardiac failure. The system functions primarily as a gas exchange center that oxygenates the patient's blood while removing carbon dioxide. ${ }^{1}$

ECMO is commonly used in the pediatric and neonatal populations, but its use in adults has increased. In adults, ECMO is typically used in cases of severe respiratory failure or following the failure to wean from cardiopulmonary bypass after cardiac surgery. ECMO is used as supportive care in situations where there is a potentially reversible process. Neurologic injury, such as anoxic injury, hemorrhage, and infarction, occurs in half of the patients treated with ECMO. ${ }^{2}$ Imaging assessment of these patients can be performed with CT imaging, as MR imaging cannot safely be used because of the ECMO equipment. $^{3}$

The utility of imaging in patients on ECMO has been reported in the literature, but there is little information describing the imaging appearance of intracranial CTA in patients on ECMO. We describe the different types of ECMO and provide a case study illustrating the conundrum associated with imaging these patients. In addition, we provide suggestions for optimizing CTA imaging of the head and neck in patients on ECMO.

Received September 16, 2016; accepted after revision November 6

From the Department of Radiology, Keck School of Medicine, University of Southern California, Los Angeles, California.

Please address correspondence to Jay Acharya, MD, Department of Radiology, University of Southern California, Keck School of Medicine, Healthcare Consultation Center II, 1520 San Pablo St, Suite L1600, Los Angeles, CA 90033; e-mail: Jay.Acharya@med.usc.edu

http://dx.doi.org/10.3174/ajnr.A5060

\section{ECMO}

The ECMO circuit comprises a pump, blender, membrane oxygenator, control console, heater and cooler, and the 2 cannulas. The pump distributes the blood though the system. The blender combines oxygen with carbon dioxide (typically, 95\% oxygen and $5 \%$ carbon dioxide). The heater and cooler function to regulate the temperature of the blood before it is reintroduced to the body. The cannulas are the output and input elements to the patient's circulatory system. ${ }^{4}$ Patients on ECMO are routinely placed on anticoagulation to prevent thrombus formation within the ECMO circuit.

There are 2 primary types of ECMO systems: venoarterial (VA) and venovenous (VV). ${ }^{5}$ Venous blood is removed from the circulation and bypassed to an external membrane oxygenation system. The oxygenated blood is then reintroduced into either the venous circulation or the arterial circulation.

Venoarterial ECMO is used in patients with both respiratory and cardiac failure. Venous blood is delivered to the external system and is oxygenated. ${ }^{6}$ The oxygenated blood is then introduced into the arterial system, typically with a high flow rate, which operates in lieu of a poorly functioning left ventricle. The venoarterial system is subdivided into a central and peripheral designation. The central type refers to arterial input of the ECMO cannula in the mediastinum, whereas for peripheral VA ECMO, the cannula is placed into a peripheral artery. Central VA ECMO involves placement of the return cannula into the aorta and the draining cannula into the right atrium via an open sternum. For the peripheral variant, the common femoral artery is the most common site for the return cannula, with the cannula tip advanced to the level of the common iliac artery or the inferior abdominal aorta. The draining cannula is often inserted into the ipsilateral com- 


\section{Peripheral Veno-arterial ECMO}

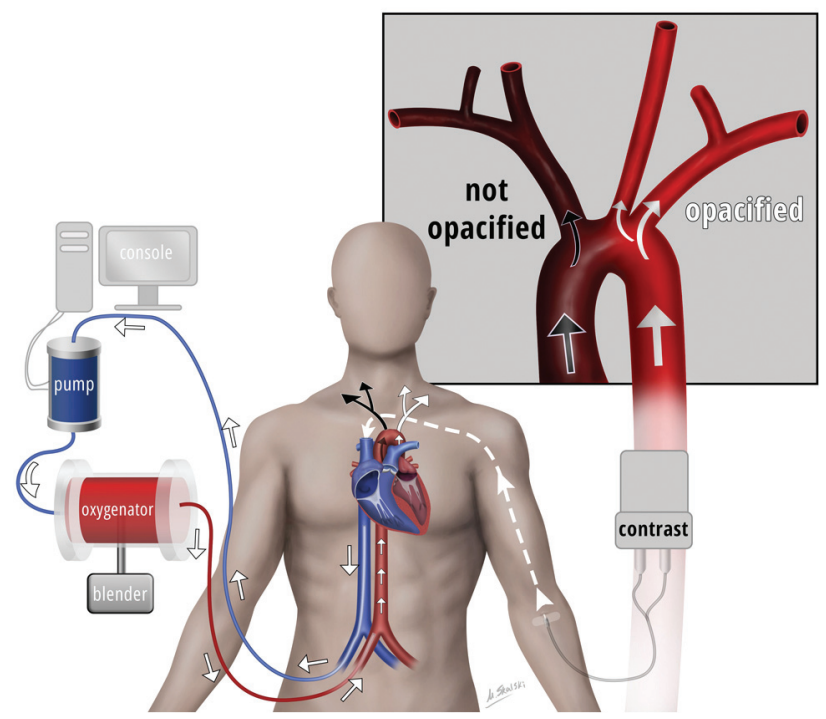

FIG 1. In the peripheral venoarterial ECMO system, the draining cannula tip is near the right atrium and the return cannula tip is in the superior right common iliac artery. When contrast (white arrows) is injected into the left antecubital fossa intravenous line, it travels toward the heart. Once it enters the right atrium, a large proportion of the contrast is sucked out through the draining cannula, then passes through the ECMO circuit, and subsequently returns into the arterial system with a high rate of retrograde flow through the descending aorta. Residual left ventricular function pumps unopacified/poorly opacified blood (black arrows) into the ascending aorta, where there is convergence with the contrast-opacified blood coming up the descending aorta. Naturally, the unopacified blood (black arrows) will preferentially fill the brachiocephalic artery and, thus, the right vertebral artery and right ICA, whereas the left common and left subclavian arteries will be preferentially contrast opacified (white arrows).

mon femoral vein, with its tip advanced to near the inferior vena cava (Fig 1$)^{7}$

Venovenous ECMO is used in patients with respiratory failure and serves to bypass the pulmonary system. The blood is returned to the right side of the heart, and no mechanical circulatory support is provided. In these patients, the native left ventricular function delivers the externally oxygenated blood to the body. VV ECMO also has several configurations. A femoroatrial configuration in which the draining cannula enters the common femoral vein, with its tip terminating below the hepatic veins, is often used. The return cannula enters the internal jugular vein, and its tip terminates near the superior cavoatrial junction (Fig 2). ${ }^{7} \mathrm{Al}-$ ternative VV ECMO configurations include the femorofemoral type as well as the dual-lumen single ECMO cannula. The femorofemoral variant introduces the draining cannula into the common femoral vein, with its tip terminating near the inferior vena cava and a return cannula inserted into either the same common femoral vein or the contralateral common femoral vein, with the tip terminating near the right atrium. ${ }^{6}$ The dual-lumen single cannula is generally introduced into the right internal jugular vein and advanced to the inferior vena cava. Alternatively, the dual-lumen cannula may be introduced into the common femoral vein, with the tip terminating near the superior vena cava. ${ }^{4}$

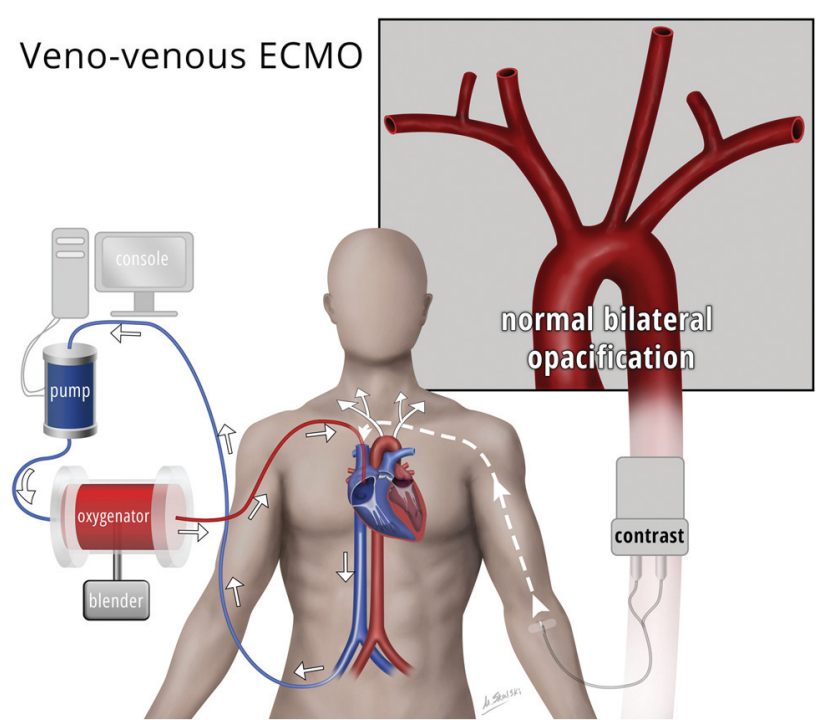

FIG 2. In the venovenous ECMO system, the draining cannula most commonly enters the common femoral vein with its tip in the inferior vena cava, near the level of the diaphragm. The return cannula is generally placed via the right internal jugular vein, with its tip in the right atrium or in the inferior aspect of the right superior vena cava. In this configuration, asymmetric arterial opacification of the head and neck will not occur, as there is preservation of the native left ventricular function. The contrast bolus density and the timing of the contrast bolus, however, will be altered.

\section{Case Example}

A previously healthy 45-year-old woman suddenly collapsed at home after 3 days of cough and shortness of breath. Emergency medical services arrived on the scene, finding the patient in ventricular tachycardia. Electrical cardioversion was performed in the field. The patient was intubated, but progressively decompensated en route to the hospital. Upon arrival, the patient required resuscitation for 75 minutes, with intermittent return of spontaneous circulation. She was placed on venoarterial ECMO. The patient's left ventricular ejection fraction was $11 \%$. During the next few days, the patient remained comatose. Multiple noncontrast CT examinations of the head were performed, all of which were negative for CT evidence of acute infarction.

Per neurosurgical request, the patient subsequently underwent CTA of the head. On the CTA, there was decreased opacification of the right internal carotid artery and right vertebral artery compared with the left ICA and left vertebral artery (Figs 3 and 4). The appearance suggested that the asymmetric vascular opacification was caused by alterations in the distribution of contrast produced by VA ECMO. This case illustrates the need for a detailed history and knowledge of typical hemodynamic changes produced by ECMO so the radiologist can properly protocol and provide an accurate interpretation of subsequent CTA imaging.

\section{DISCUSSION}

When contrast material is injected intravenously, the venous draining cannula will siphon the contrast-opacified blood, where it will be transported through the ECMO device and reintroduced systemically through the return cannula. In VA ECMO, contrastopacified blood will enter the arterial system at a high rate and flow into the descending aorta in a retrograde fashion. Therefore, if using a standard bolus-tracking technique, with an ROI placed 


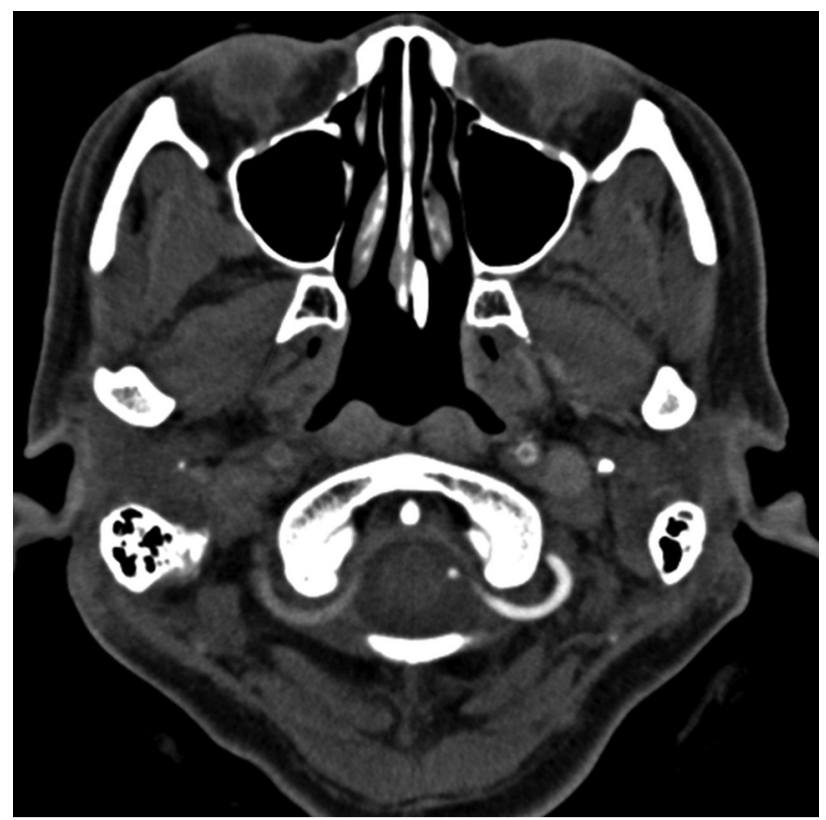

FIG 3. CTA of the head. CTA of the head demonstrates asymmetric opacification of the V3 segments of the bilateral vertebral arteries. Contrast avidly opacifies the left V 3 segment because of retrograde flow of contrast through the descending aorta and opacification of the left subclavian and left vertebral arteries. This is not the sequela of arterial thrombosis or dissection. Residual left ventricular function pumps poorly opacified blood preferentially into the brachiocephalic artery and the right vertebral artery.

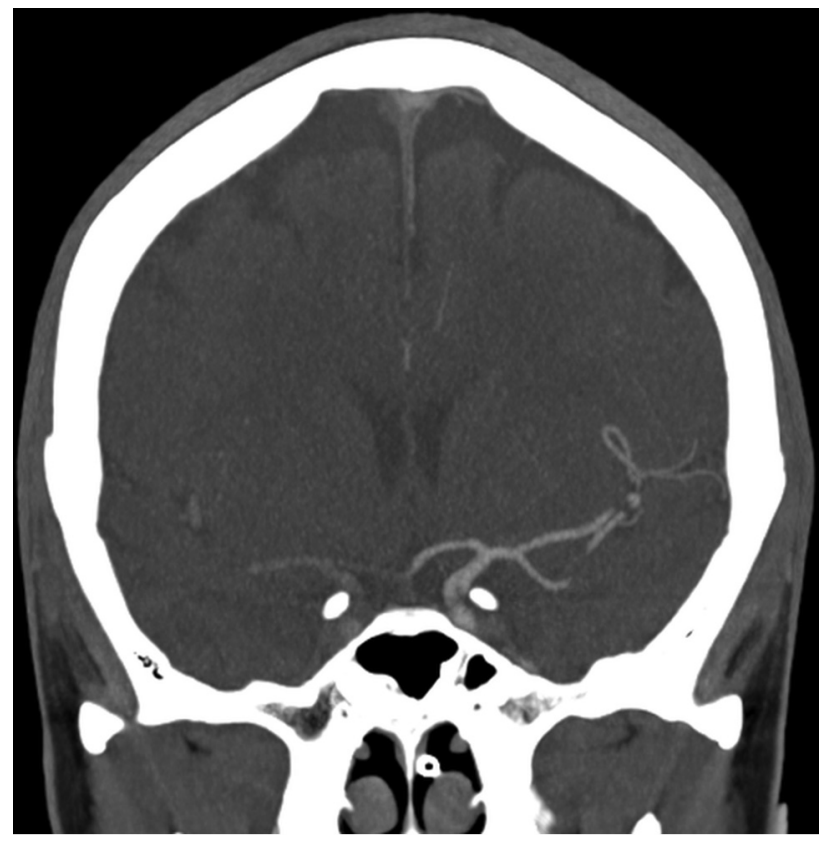

FIG 4. CTA of the head. Coronal 7.0-mm MIP image demonstrates asymmetric opacification of the distal ICA, anterior cerebral artery, and MCA. Contrast avidly opacifies the distal left ICA, left Al segment, and left MCA. This is not the sequela of an arterial thrombosis or dissection. Residual left ventricular function pumps poorly opacified blood preferentially into the brachiocephalic artery and to the right ICA and is the cause of this asymmetric appearance.

in the ascending aorta, it may not be possible to correctly time the contrast bolus necessary to correctly perform CTA of the head. The retrograde flow of blood in the aorta depends on 2 factors: the flow rate in the ECMO system and the degree of residual left ventricular function. Patients with little or no left ventricular function will have more retrograde flow through the aorta from the ECMO system containing contrast-opacified blood, which will reach the aortic arch and the great arterial vessels. Effective retrograde perfusion of the entire aortic arch and great vessels requires complete bypass of the heart in patients with zero left ventricular function and a closed aortic valve. ${ }^{8}$ When the patient has some preservation of left ventricular function and ejection fraction, unopacified blood that travels antegrade from the left ventricle into the aortic arch will mix with the contrast-opacified blood traveling in a retrograde manner from the ECMO system. The unopacified blood from the left ventricle will preferentially flow into the brachiocephalic artery and, subsequently, the right common carotid and right vertebral arteries. Opacified blood flowing from the ECMO system, retrograde through the aorta, will preferentially fill the left subclavian artery and left common carotid artery. This creates asymmetric opacification of the great arterial vessels of the head and neck. This principle is illustrated in our case, in which there is diminished contrast opacification in the right ICA and right vertebral artery relative to the left ICA and left vertebral artery because the patient had residual left ventricular function.

Neurologic sequelae, such as $\mathrm{SAH}$, ischemic borderzone infarctions, hypoxic-ischemic encephalopathy, and brain death, often occur in adult patients treated with ECMO. ${ }^{2}$ More recently, cerebral microhemorrhages have also been shown to be associated with patients treated with ECMO. ${ }^{9}$ Some of these findings may be more difficult to assess using CT imaging, but it is important for the radiologist to be aware of the association between ECMO and these neurologic sequelae, especially because the conventional ECMO equipment is not safe for MR imaging.

\section{Optimizing Technique}

Cardiocirculatory function in patients on VV ECMO is relatively normal, with dynamic contrast-enhanced CT imaging comparable with that seen in healthy people. ${ }^{10}$ The timing of contrast opacification of the aorta may be variable in these patients, but the contrast bolus should be similar to that seen in healthy patients. Thus, the use of a bolus-tracking system is generally adequate in this patient population.

The typical flow rate for VA ECMO is between $4-6 \mathrm{~L} / \mathrm{min}$ in adults and is adjusted based on the patient's hemodynamic status. A flow rate between $50-100 \mathrm{~mL} / \mathrm{kg} / \mathrm{min}$ may also be used, but these rates are adjusted on a case-by-case basis, depending on the clinical status of the patient.

The literature describes techniques to optimize CT pulmonary angiography for patients on VA ECMO. Lee et $\mathrm{al}^{4}$ recommended reducing the ECMO flow rate to $500 \mathrm{~mL} / \mathrm{min}$ for $15-25$ seconds, injecting the intravenous contrast, and placing the bolus-tracking ROI over the main pulmonary artery. Bolus tracking for assessment of the pulmonary vasculature is typically placed over the main pulmonary artery in patients not on ECMO as well. ${ }^{11}$ With a decreased flow rate, the contrast will opacify the pulmonary arteries. ${ }^{10}$ Lee et al ${ }^{4}$ suggested that CTA acquisition may also be manually triggered when the ROI demonstrates a peak or plateau level of opacification in the main pulmonary artery. Al- 
though these principles have been described as effective techniques in CT pulmonary angiography, they do not apply to CTA of the head.

CTA imaging of the head can be optimized in patients on VA ECMO by modifying the suggestions used for evaluation of the pulmonary arteries. Decreasing the flow rate in VA ECMO will reduce the extent of the retrograde flow in the aorta. In turn, this will diminish the mixing of opacified and unopacified blood within the aortic arch and great arterial vessels of the neck. Alternatively, the ECMO may be put on hold altogether for the duration of the scan if the patient is able to tolerate this. Temporarily restoring the physiologic antegrade flow of blood through the ascending aorta and aortic arch as the primary source of flow to the great vessels permits the use of a bolus-tracking system. Manual triggering of the CTA head could also be done after reduction or a pause in the VA ECMO flow rate.

However, in many cases, including the case we describe above, the patient may not be able to be weaned off of their ECMO or tolerate even temporary changes to the flow rate to optimize CTA imaging. Malinzak and Hurwitz ${ }^{12}$ described a case in which ECMO flow rates could not be reduced in a patient on VA ECMO. The patient received a 1-mg dose of epinephrine at the same time as the contrast injection during CT pulmonary angiography. The ECMO was stopped for 12 seconds during the scanning procedure without reported adverse outcome and resulted in restoration of the physiologic flow dynamics and an adequate study quality. Some patients also may not have sufficient native left ventricular function to pump blood to the great vessels if the VA ECMO flow rate is reduced. Having an existing echocardiogram and cardiology assessment of the left ventricular function is essential in planning CTA head imaging for patients on VA ECMO.

\section{CONCLUSIONS}

Hemodynamic changes produced by ECMO can confound accurate interpretation of CTA head imaging. Radiologists should understand the principles, physiology, and types of ECMO. This knowledge, as well as techniques used for evaluating the pulmonary circulation, may allow for optimized CTA imaging of the intracranial vasculature.

\section{REFERENCES}

1. Zapol WM, Snider MT, Hill JD, et al. Extracorporeal membrane oxygenation in severe acute respiratory failure. A randomized prospective study. JAMA 1979;242:2193-96 CrossRef Medline

2. Mateen FJ, Muralidharan R, Shinohara RT, et al. Neurological injury in adults treated with extracorporeal membrane oxygenation. Arch Neurol 2011;68:1543-49 CrossRef Medline

3. Lidegran MK, Frenckner BP, Mosskin M, et al. MRI of the brain and thorax during extracorporeal membrane oxygenation: preliminary report from a pig model. ASAIO J 2006;52:104-09 CrossRef Medline

4. Lee S, Chaturvedi A. Imaging adults on extracorporeal membrane oxygenation (ECMO). Insights Imaging 2014;5:731-42 CrossRef Medline

5. Brodie D, Bacchetta M. Extracorporeal membrane oxygenation for ARDS in adults. N Engl J Med 2011;365:1905-14 CrossRef Medline

6. Sidebotham D, McGeorge A, McGuinness S, et al. Extracorporeal membrane oxygenation for treating severe cardiac and respiratory disease in adults: Part 1-overview of extracorporeal membrane oxygenation. J Cardiothorac Vasc Anesth 2009;23:886-92 CrossRef Medline

7. Platts DG, Sedgwick JF, Burstow DJ, et al. The role of echocardiography in the management of patients supported by extracorporeal membrane oxygenation. J Am Soc Echocardiogr 2012;25:131-41 CrossRef Medline

8. Soeter JR, Smith GT, Anema RJ, et al. Distribution of oxygenated blood in femoral and brachial artery perfusion during venoarterial bypass in primates. J Thorac Cardiovasc Surg 1973;65:825-29 Medline

9. Le Guennec L, Bertrand A, Laurent C, et al. Diffuse cerebral microbleeds after extracorporeal membrane oxygenation support. $A m \mathrm{~J}$ Respir Crit Care Med 2015;191:594-96 CrossRef Medline

10. Liu KL, Wang YF, Chang YC, et al. Multislice CT scans in patients on extracorporeal membrane oxygenation: emphasis on hemodynamic changes and imaging pitfalls. Korean J Radiol 2014;15:322-29 CrossRef Medline

11. Wittram C. How I do it: CT pulmonary angiography. AJR Am J Roentgenol 2007;188:1255-61 CrossRef Medline

12. Malinzak M, Hurwitz LM. Pulmonary CTA in the setting of venoarterial extracorporeal membranous oxygenation. SOMATOM Sessions Online: The Magazine for Computed Tomography 2015;35:54-55 